\title{
Automation Interfaces of the Orion GNC Executive Architecture
}

Jeremy Hart Orion Flight Dynamics Team GNC Automation and Autonomy Lead 281-483-0001 ieremyihart@nasa.cov

AIAA GNC Conference. August 2009
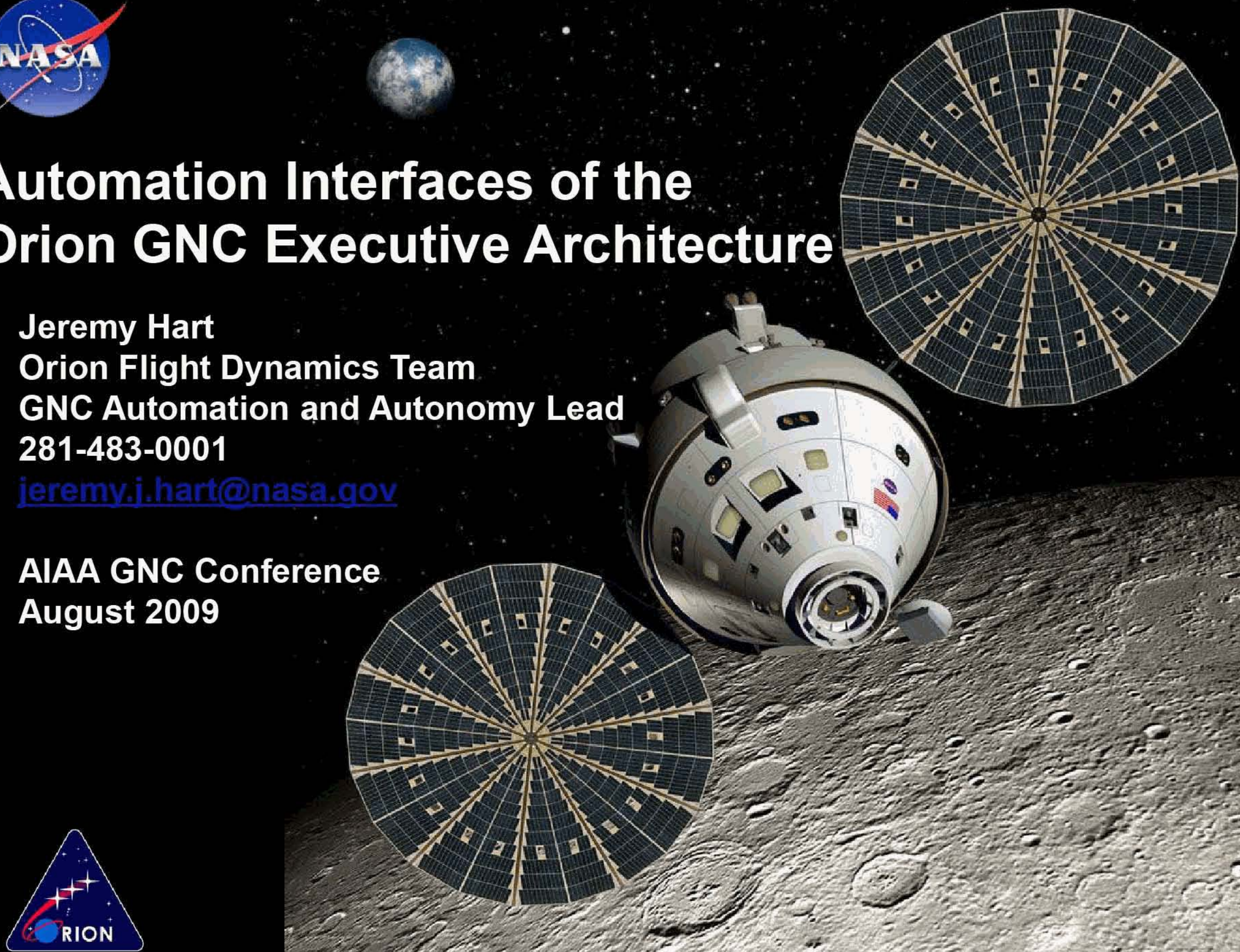

5

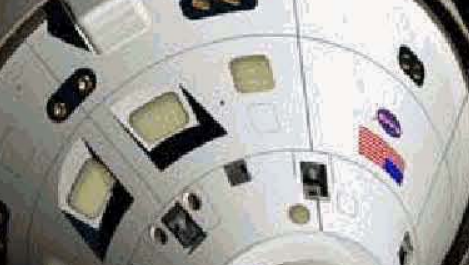


- Orion Background

- Shuttle / Orion Automation Comparison

- Orion Mission Sequencing

- Orion Mission Sequencing Display Concept

- Status and Forward Plans 


\section{Orion Background}




\section{Constellation Program Definitions: Automation and Autonomy}

\section{- Automated}

- Control or execution of a system or process without human intervention or commanding.

- Function performed via ground and/or onboard software interaction.

- This does not exclude the possibility of operator input, but such input is explicitly not required for an automated function.

\section{- Autonomous}

- Capable of operating independent of external communication, commands or control (i.e. commands from mission control on Earth).

- Can involve crew and software in nominal and contingency operations.

- GN\&C architecture is designed for automated operation

- New capabilities include: Automated Rendezvous and Docking, Automated Deorbit

- Automated functionality allows for increase spacecraft autonomy from ground support, allowing:

- Uncrewed missions

- Increased capability for loss-of-comm scenarios

- Reduced requirements for ground support 


\section{Orion Mission Overview}

Orion Project

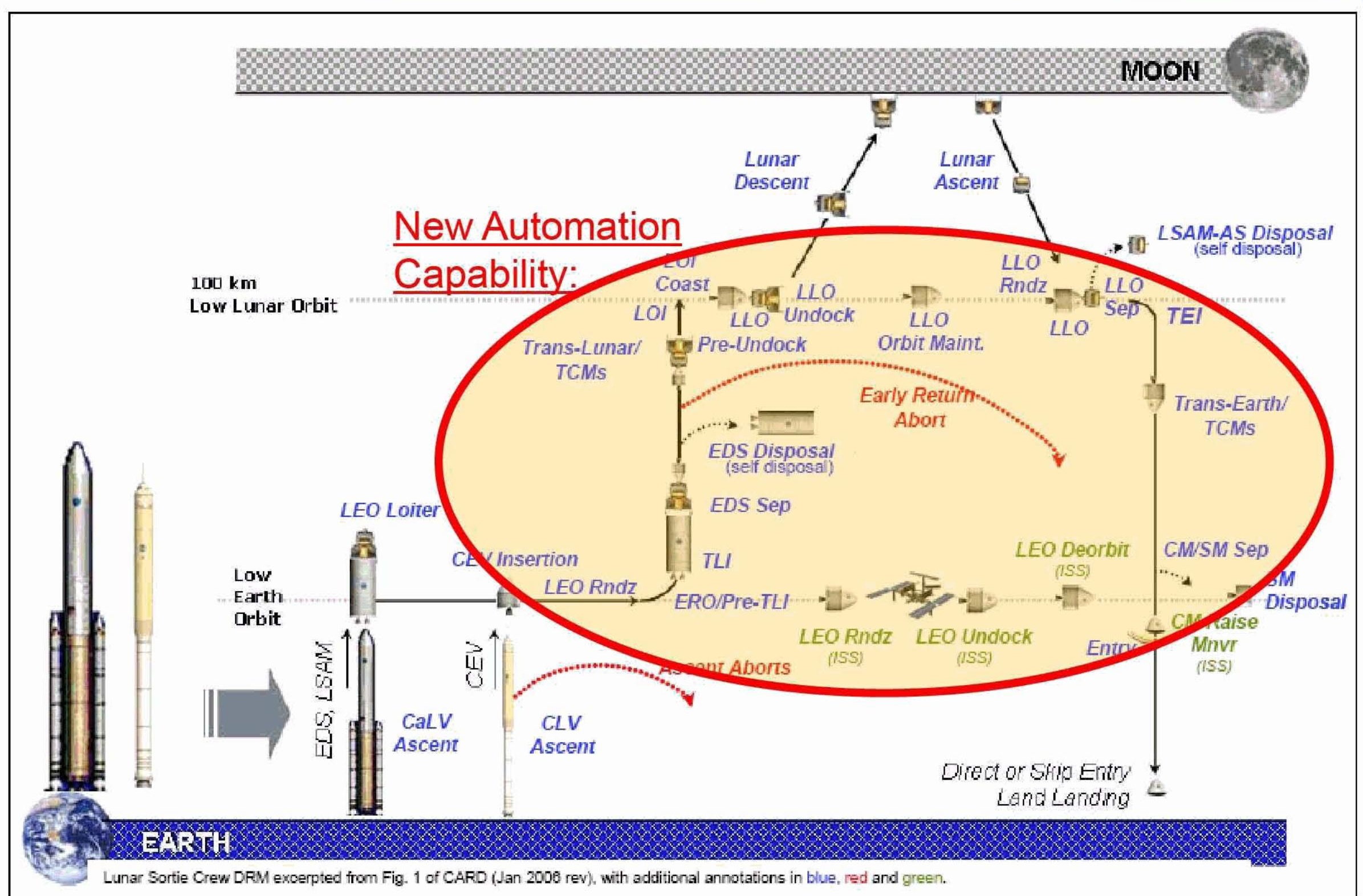




\section{GN\&C Automation Design Principles}

- GN\&C is architected for automated operation with AND without crew

- Automated sequencing and configuration of GN\&C events

- Nominal

- Pre-defined Contingencies

- This does not imply that all functionality necessary to execute the mission is fully automated.

- Orion automation is designed for gradual adoption and future evolution of functionality

- GN\&C interfaces are based on crew and ground involvement, including:

- Situational awareness and manual reconfiguration of GN\&C functionality

- Authority-To-Proceed prior to critical events

- Inhibit/re-enable and terminate automated functionality

- Manual downmodes/contingencies (including Manual Piloting)

Orion GN\&C architecture is designed for evolutionary incorporation of automated functionality.

Crew and ground interaction and manual commanding capabilities are provided. 


\section{Shuttle / Orion Automation Comparison}




\section{- Automated functionality changes the role of the crew}

- The nominal on-board operation shifts from manual configuration to primarily monitoring and situational awareness

- GN\&C software is capable of automated configuration and sequencing

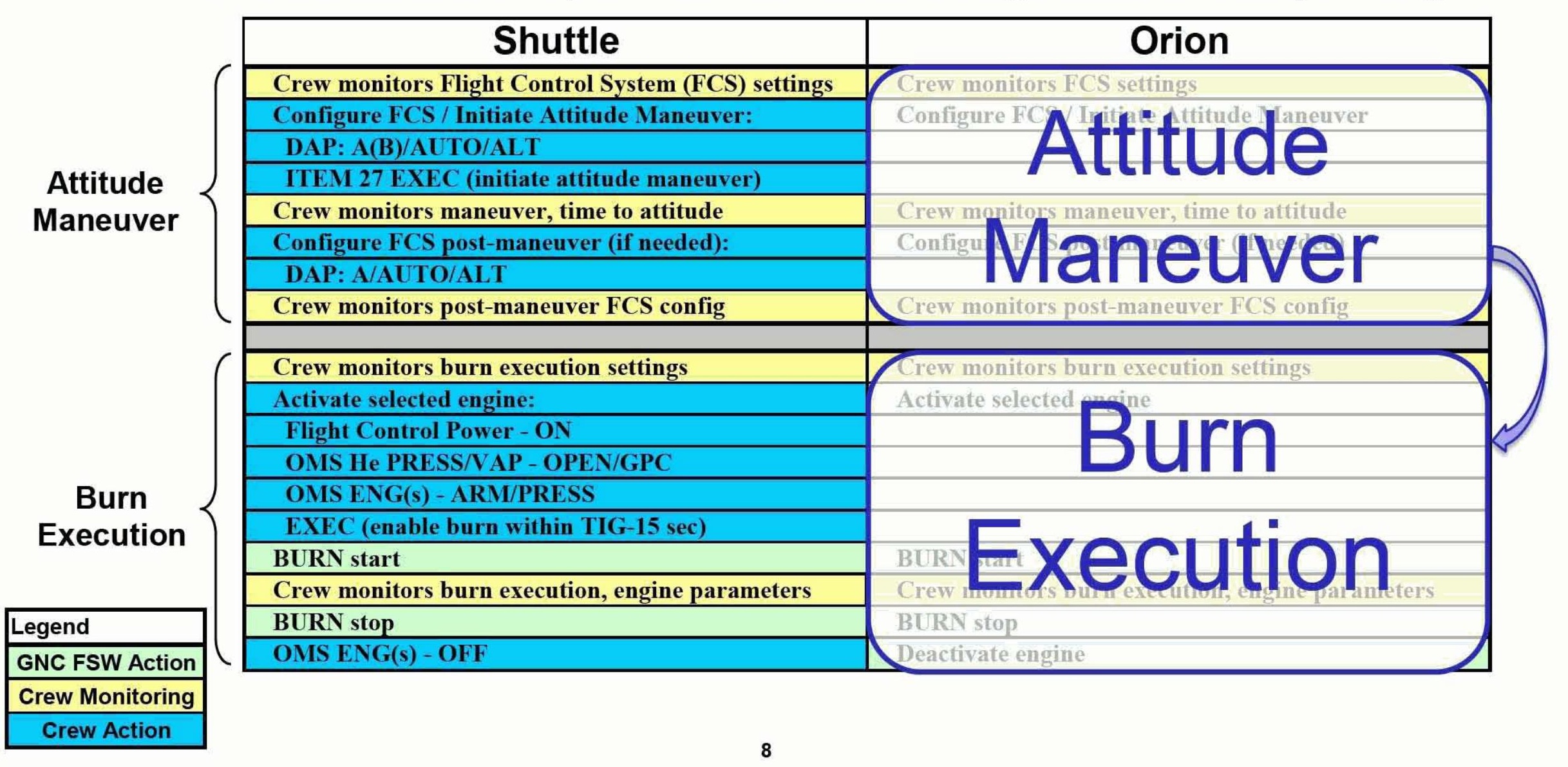




\section{Orion Mission Sequencing}




\section{Mission Sequencing Hierarchy}

- GN\&C Subsystem is responsible for:

- Subsystem configuration

- Sequencing of GN\&C Activities, e.g. Attitude Maneuver, Burn Execution

- GN\&C Activities coordinate the software components of the GN\&C subsystem

- Transitions based on parameters internal to GN\&C
GN\&C Activities

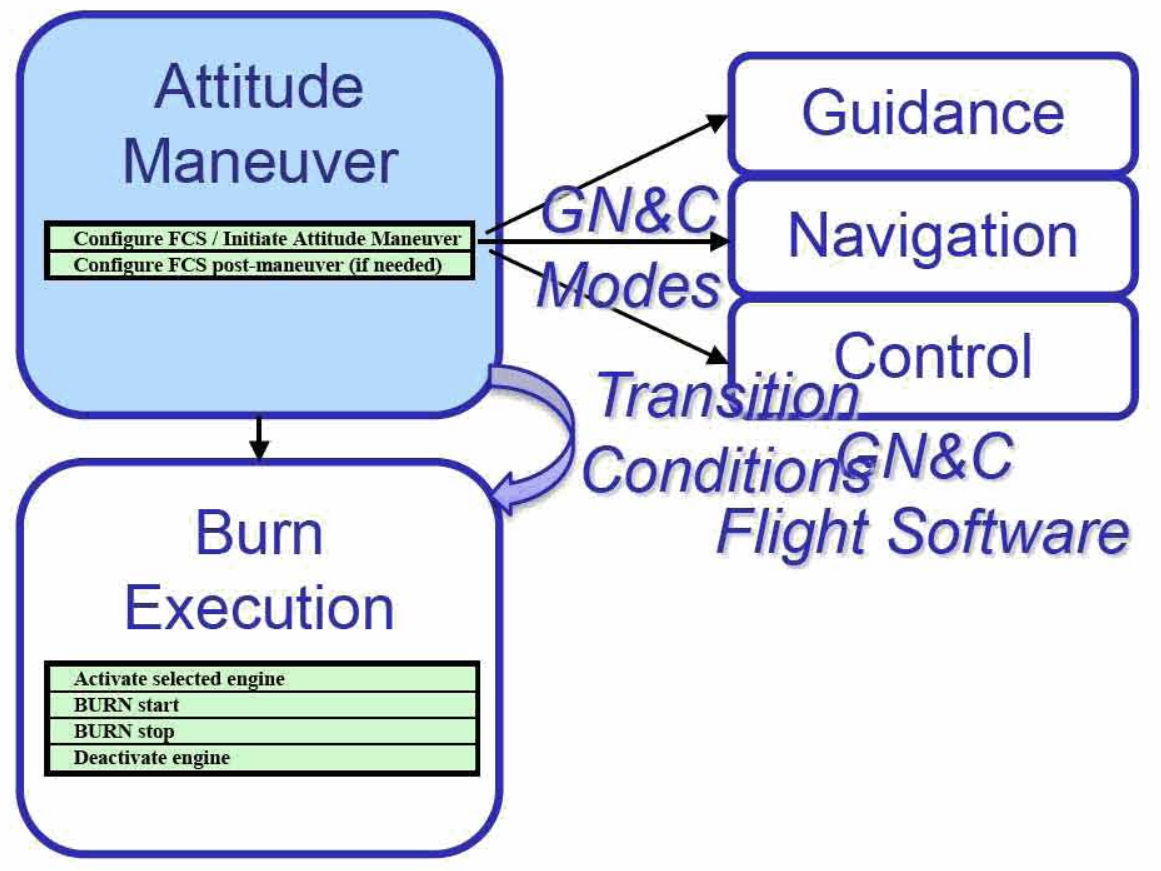




\section{Mission Sequencing Hierarchy}

Orion Project

\begin{tabular}{ll|ll}
$\begin{array}{l}\text { Phases } \\
\sim \text { Shuttle OPS }\end{array}$ & $\begin{array}{l}\text { Segments } \\
\sim \text { Shuttle Major Modes }\end{array}$
\end{tabular} GN\&C Activities $\quad$ GN\&C Modes

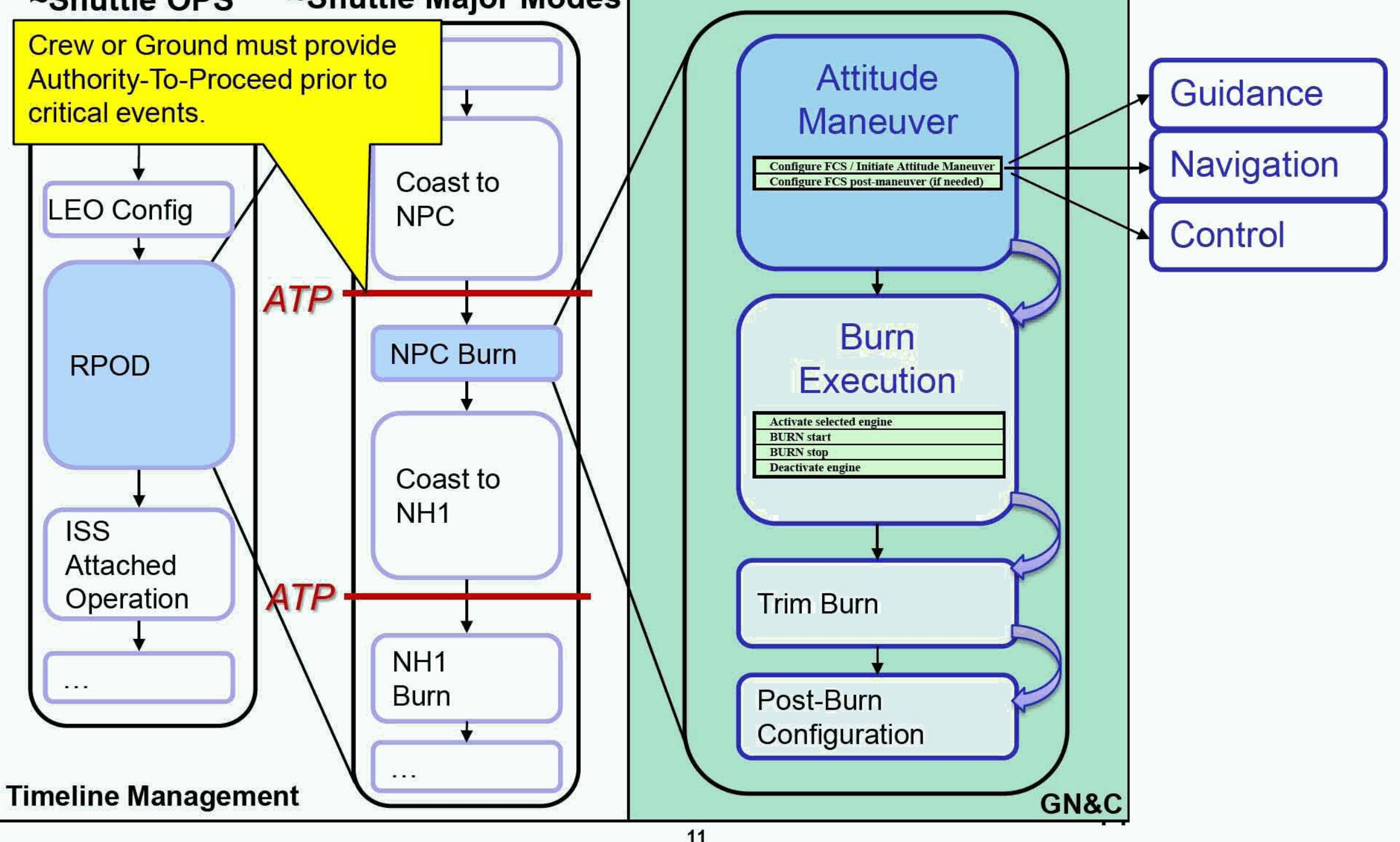




\section{Mission Sequencing Hierarchy}

Orinn Drnient

Phases -Shuttle OPS
Segments

Shuttle Major Modes
GN\&C Activities
GN\&C Modes

Phase, Segment, Activity, Mode = PSAM

Defines GN\&C configuration and sequencing throughout the mission
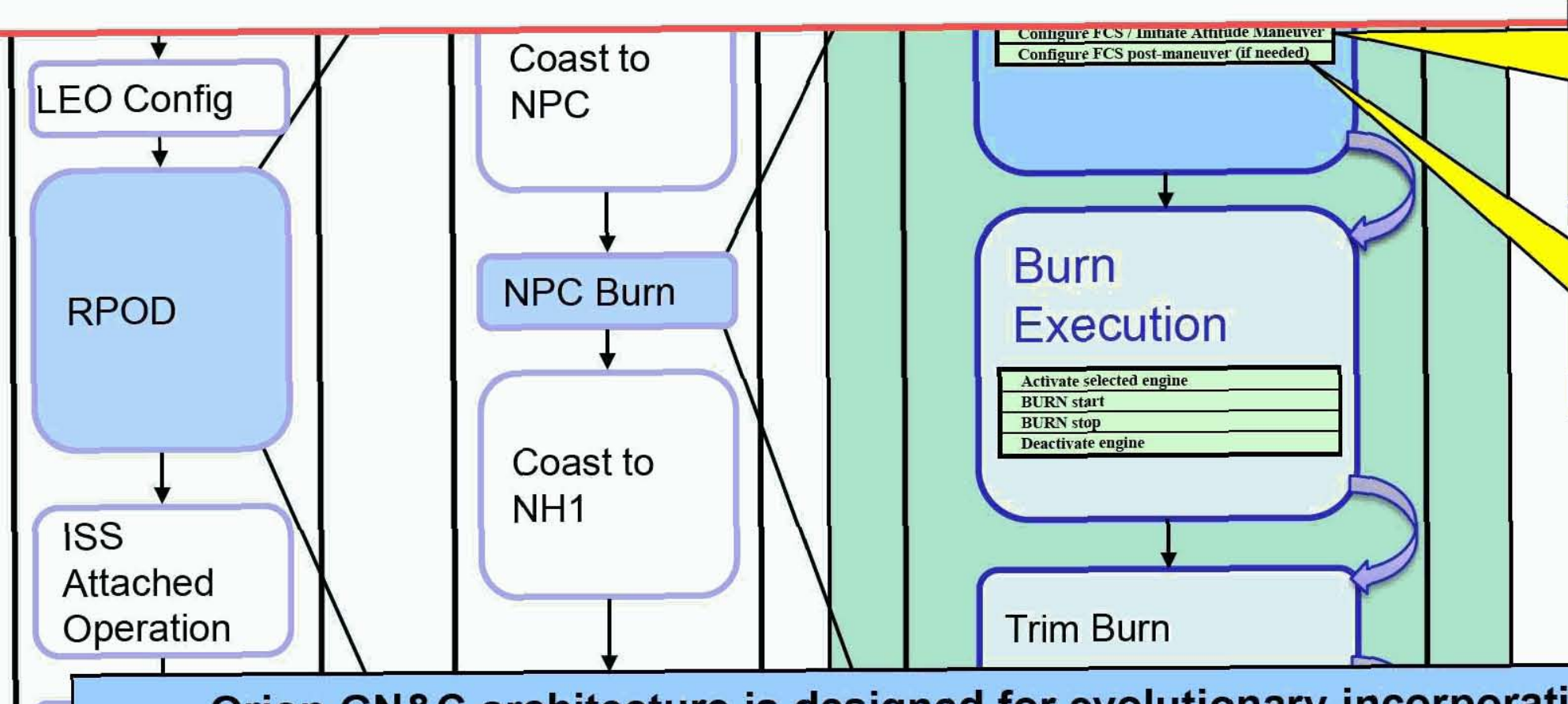

Crew Monitors: -Attitude, rate -FCS config -Trajectory

(Optional) Manual Updates: -Alternate FCS configuration

Orion GN\&C architecture is designed for evolutionary incorporation of automated functionality.

Crew and ground interaction and manual commanding capabilities are provided. Timeline Management 


\section{Orion Mission Sequencing Display Concept} NOTIONAL 


\section{Introduction: Automated Sequencing Display}

Orion Project

- Developed to increase understanding of automated sequencing as it stands in the design

- Use of Mission Segments and GNC Activities to monitor automated functionality

- Proposes use of common displays for manual commanding and automated sequencing

- Common and consolidated interface with Mission Segments and GNC Activities

- Combine automated sequencing into one consolidated area

- Treat Mission Segment level ATP and GNC Activity level inhibit/enable with a transparent interface

- Describe manual interaction with automated sequencing

- Nominal Sequencing

- Reconfiguration of Sequence

- Manual / Contingency Operations 


\section{Orion Sequencing Display Concept (DRAFT)}

\section{Orion Project}

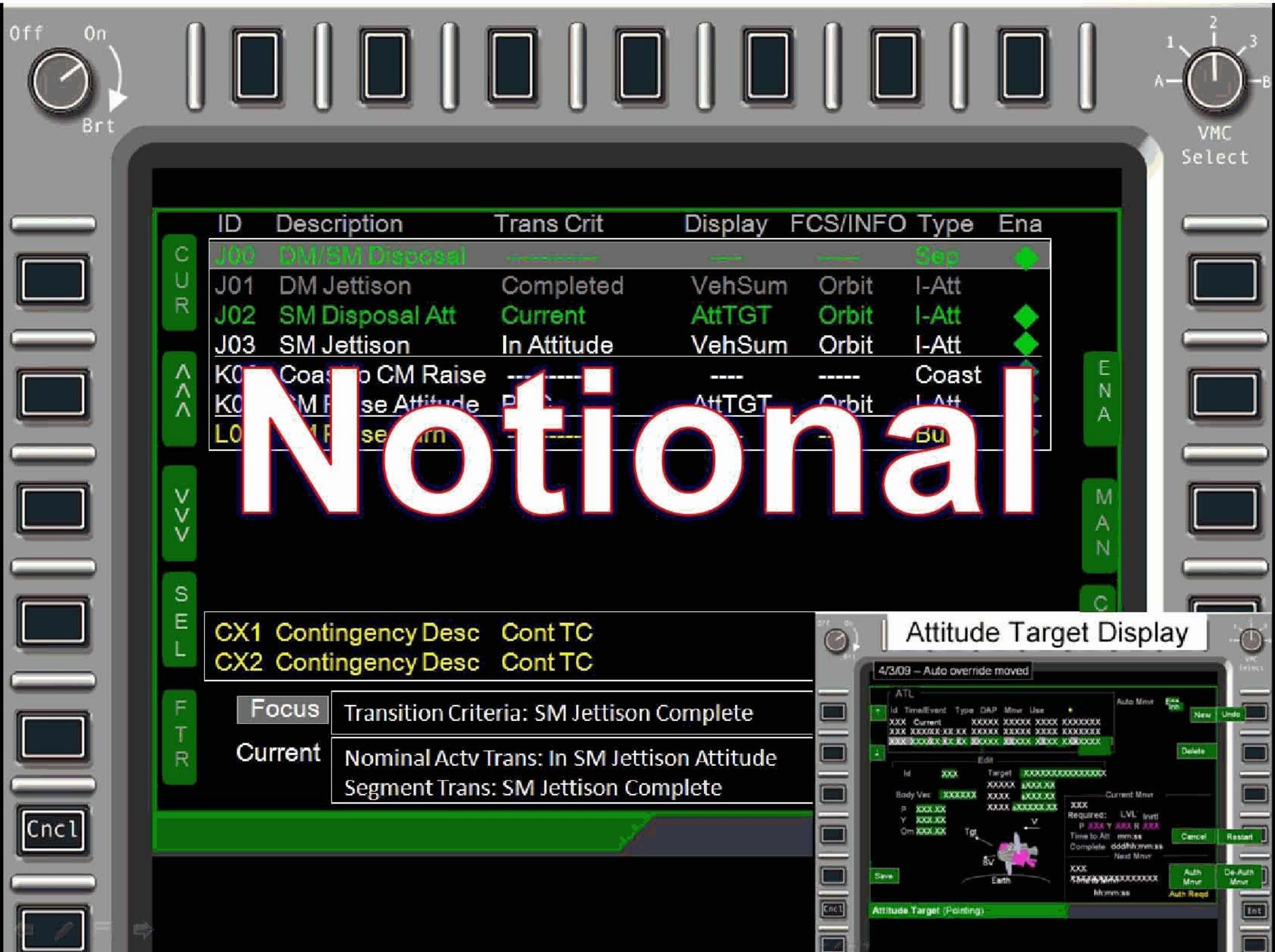




\section{Nominal Automated Execution}

- Summary info for Nominal Segments/Activities on a compact navigable list

- Activity/Segment ID

- Source (e.g. TM, GNC, Other Subsystems, etc.)

- Description - text to help the user Situational Awareness, as detailed as makes sense

- Time / Event Trigger

- ATP/Enable indication

- Contingency Segment (for the current activity)

- Navigation of summary list:

- Select/Inspect (Bring into focus, display additional details)

- Navigate up/down the list

- Filter the list based type

- Execute selected 


\section{Reconfiguration or Off-Nominal Operations}

- Editing Existing Segment/Activities

- Detailed edits available via individual subsystem displays

- Sequencing information (when selected):

- Activity Transition criteria (View, edit, save)

- Contingency Segment

- Replanning/contingencies

- Manually command to a new segment

- Command contingency segment

- Exit automated sequence:

- Generic Coast/Burn capabilities

- Individual actions available via subsystem displays

- Resume automated sequence 


\section{Status and Forward Plans}


- Mission Sequencing display concept was accepted part of the Orion 'Pass 1' display suite

- Initial crew preference is to use GNC displays for nominal operations and use the Mission Sequencing display for off-nominal reconfiguration

- Orion operational concepts continue to evolve with increased design maturity

- Additional crew and ground operator involvement will continue maturation process

- All display content will continue to mature via operatorin-the-loop simulations planned for 'Pass 2' development 


\section{Contributors}

- Ellis King

- Ryan Odegard

- Chris Foster

- Ray Barrington

- Theresa Spaeth

- Jerry Yencharis

- Alan Fox

- Dale Howell

- Carlos Garcia-Galan

- Pete Spehar 


\section{Questions??}




\section{Backup}


- Ascent and Entry phases of flight are time critical and thus highly automated (even for Shuttle).

- Orion requirements for automated RPOD and Deorbit result in automated capability for all major GN\&C phases.

- The requirements for reconfigurable sequences, uncrewed flight configuration, and reduced operations cost also increase Orion automation.

The Orion requirements necessitate onboard software that can automatically sequence through and execute mission events during all phases of flight. 


\section{Mission Sequencing: Phases, Segments and Activities}

- The Mission Event Plan (MEP) is broken down into highlevel Mission Phases

- Examples: Pre-launch, Ascent, LEO Configuration, LEO Loiter, RPOD Operation, ISS Attached Operation, ISS Departure, ISS Deorbit, Entry, Descent \& Landing

- Mission Phases are divided into Mission Segments

- Pre-launch Phase Example Segments: Pre-launch, Terminal Count Down

- Ascent Phase Example Segments: 1st Stage Ascent, 2nd Stage Ascent with LAS, 2nd Stage Ascent without LAS, Coast to Orbit Insertion Burn, Orbit Insertion Burn 
Orion Project

- Automated Sequencing of Mission Events (nominal)

- Transition from automated sequencing to manual mission execution

- Available for transition to manual commanding/piloting

- When automated transitions are no longer appropriate/required automated sequencing will halt in a benign state

- Automated sequences defined to manually resume automation at appropriate points

- Manual Execution of Mission Events

- Manual command Mission Segments or GN\&C Activities

- GN\&C functionality during manual execution is equivalent to functionality used in automated sequences

\section{- Manual Piloting}

- In parallel with automation for certain scenarios, e.g. final approach during early Orion missions

- Available during contingency operations

Human interaction ranges from situational awareness to manual piloting. This functionality will result in a different operational concept than Shuttle. 


\section{Automated vs. Manual}

There are several levels of automation that can be performed with the current architecture, which allows for mission-specific flexibility.

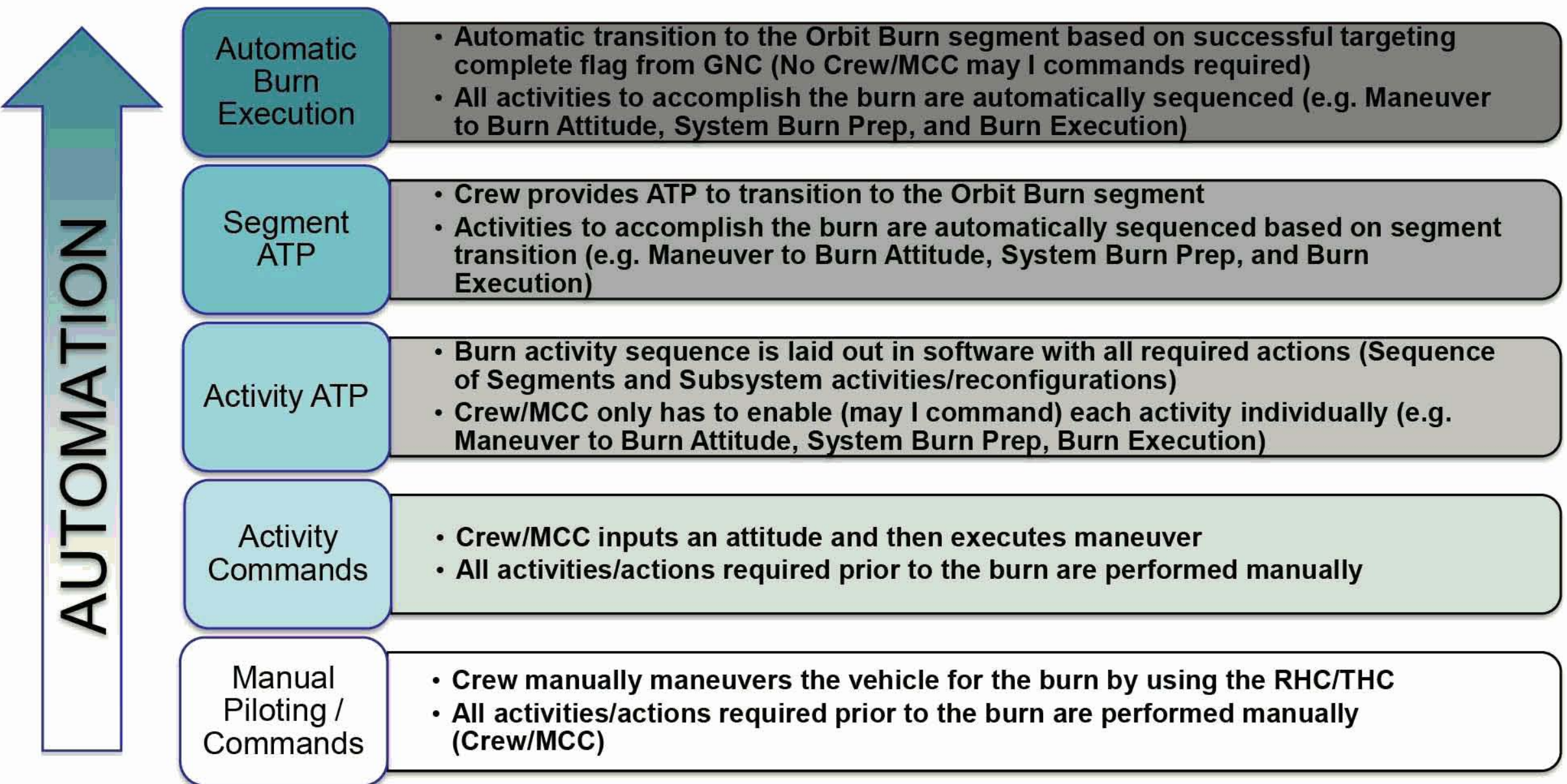




\section{GN\&C Domain Mode Commanding}

Orion Project

- Each GN\&C Activity will result in the configuration of the complete GuidanceNavigation-Control Subsystem via Mode commands sent by the GN\&C Executive

- 'IDLE' Modes used during the mission to deactivate unused flight software domains

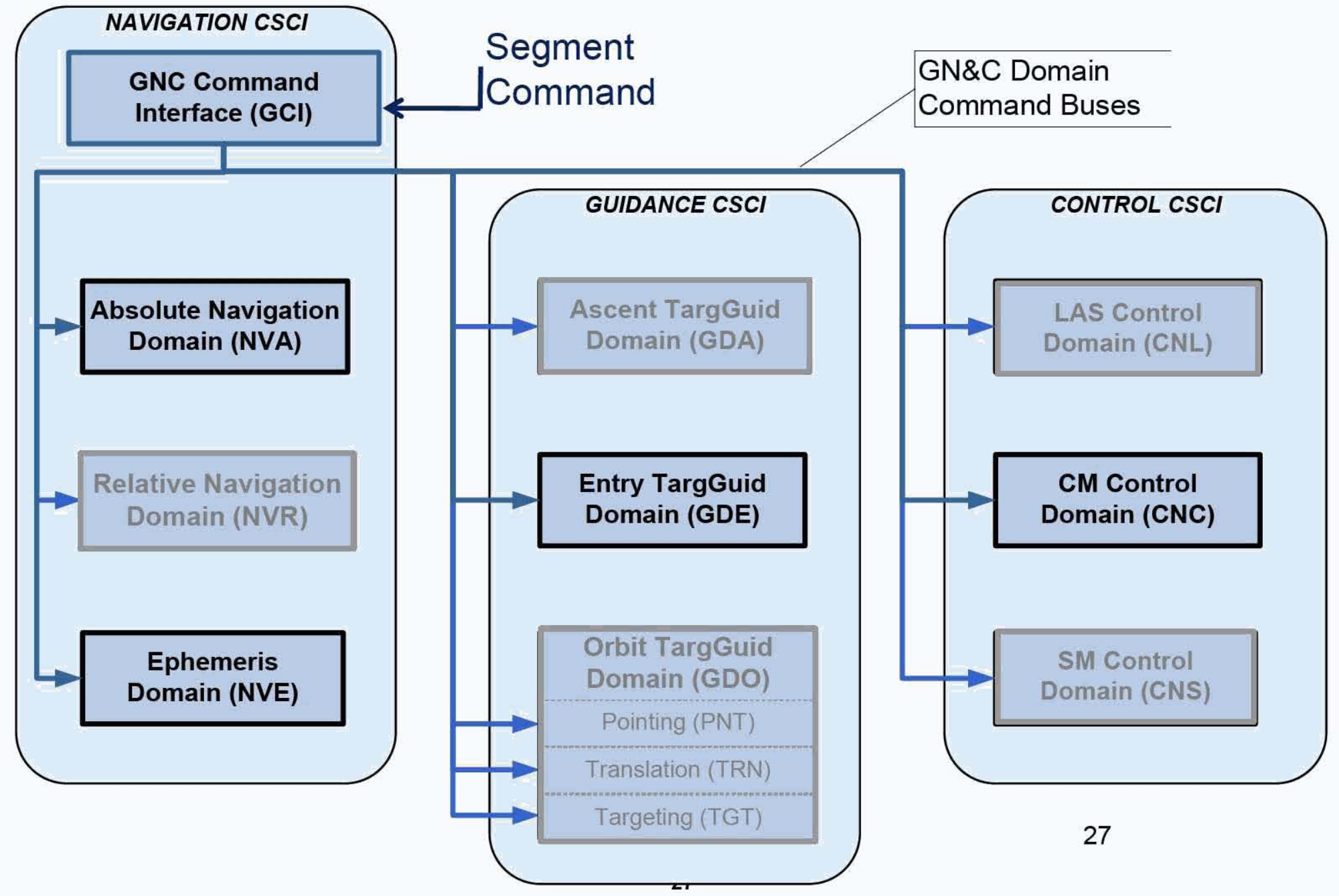




\section{Contingency Example - Backout}

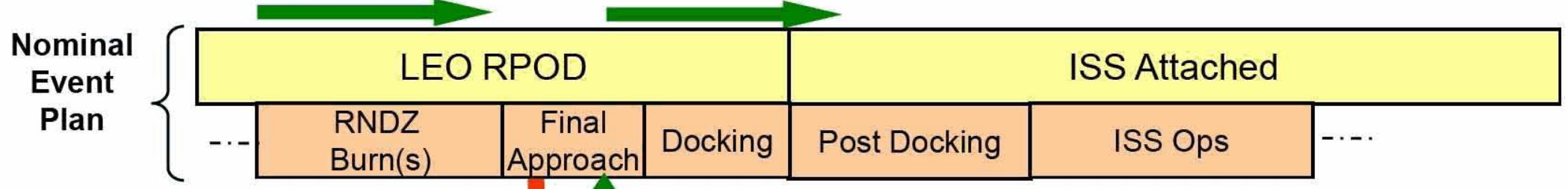

(2)

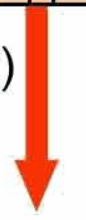

Orion Project

(1)

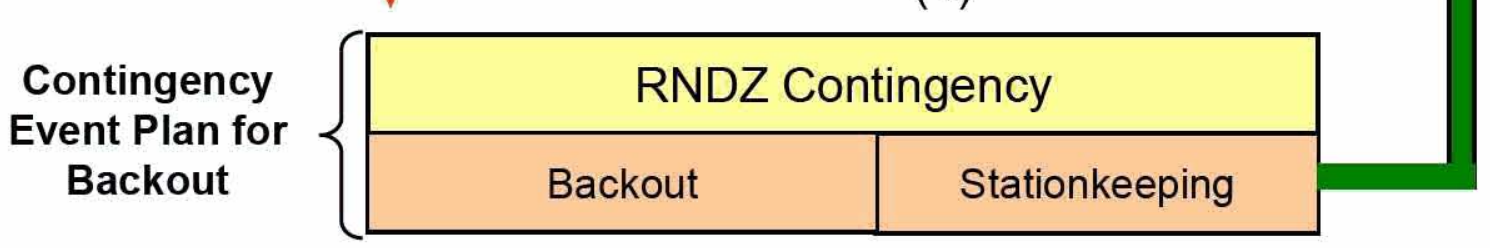

(4)

(3)

(1) Timeline Management is sequencing through the nominal mission event plan based on transition logic.

(2) GN\&C detects a problem which requires a backout (e.g. failure to capture) during the final approach segment. GN\&C sends a flag indicating the problem, which triggers Timeline Management to transition to the Contingency Event Plan for a Backout.

(3) Timeline Management switches to the Contingency Event Plan for a Backout and sequences through this new event plan based on the transition logic.

(4) If the problem is resolved, Timeline Management transitions back to the nominal flight event plan starting in the Final Approach segment when the crew/ground commands the transition.

(5) Timeline Management continues sequencing through the nominal flight event plan based on transition logic. 